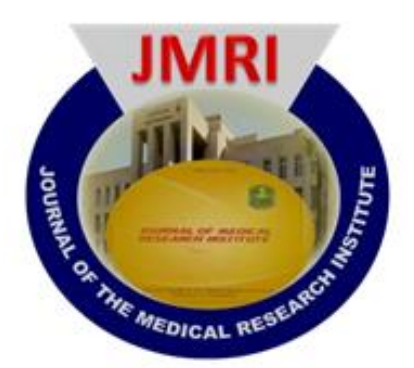

\title{
URINARY NEUOTROPHIL GELATINASE ASSOCIATED LIPOCALIN AS A DIAGNOSTIC AND A PROGNOSTIC MARKER FOR RENAL INSULT IN CIRRHOTIC PATIENTS WITH SPONTANEOUS BACTERIAL PERITONITIS
}

\author{
Mohamed Said Sayed Shater ${ }^{(1)}$, Amr Aly Abdel Moety ${ }^{(2)}$, Doaa Ibrahim Hashad ${ }^{(3)}$, Heba Seleim El- \\ Shair $^{(4)}$, Azza Abdelwahhab Ahmed ${ }^{(5)}$
}

1) Lecturer in Internal Medicine, Hepatobiliary Unit, Department of Internal Medicine, Faculty of Medicine, Alexandria University.

2) Professor of Internal Medicine, Department of Internal Medicine, Faculty of Medicine, Alexandria University

3) Professor of Clinical Pathology, Department of Clinical Pathology, Faculty of Medicine, Alexandria University

4) Professor of Internal Medicine, Department of Internal Medicine, Faculty of Medicine, Alexandria University

5) MBBCh 2009, Faculty of Medicine, Alexandria University

\begin{abstract}
:
Background: Spontaneous bacterial peritonitis (SBP) is one of the common and lethal infections in patients with liver cirrhosis. It is frequently complicated with acute kidney injury (AKI), which makes the condition worse with higher mortality. Urinary markers may have potential clinical importance in this setting. Aim: To assess the utility of urinary neutrophil gelatinaseassociated lipocalin (UNGAL), in early detection of AKI in patients with liver cirrhosis with SBP, and evaluating its prognostic role in the detection of the outcome and mortality in those patients. Materials and Methods; group (1): 30 patients hospitalized with cirrhotic liver and SBP and group(2): 30 patients with liver cirrhosis without SBP. AKI was defined according to Acute Kidney Injury Network (AKIN) criteria. uNGAL was measured. Results: AKI patients $(\mathrm{n}=21)$ had higher uNGAL levels compared to patients without AKI $(\mathrm{n}=9),(7.55 \pm 3.20$ vs. $1.23 \pm 0.78 \mathrm{ng} / \mathrm{ml}, P=0.001)$, patients with AKI persisted more than 3 days had higher uNGAL levels at diagnosis of SBP in comparison to patients who less than 3 days AKI $(8.88 \pm 1.51$ vs.4.37 \pm $1.95 \mathrm{ng} / \mathrm{ml} ; \mathrm{P}=0.005$ ), both model of end stage liver disease-sodium (MELD $\mathrm{Na}$ and uNGAL level at diagnosis of SBP are an independent predictor of 3months mortality. Conclusion: In patients with liver cirrhosis and SBP, measurement of UNGAL at time of SBP diagnosis can diagnose AKI and predict important clinical outcome, as persistency of AKI and three months mortality..
\end{abstract}

Keywords: liver cirrhosis, Spontaneous bacterial peritonitis, acute kidney injury, urinary neutrophil gelatinase-associated lipocalin (uNGAL).

\section{INTRODUCTION}

Cirrhotic patients have greater chance of developing life threatening bacterial infections including spontaneous bacterial peritonitis (SBP).(1, 2)Among complications associated with SBP; kidney injury occurs in $~ 35 \%$ of SBP cases and is considered a strong predictor of hospital mortality.(3)Renal injury 2ry to SBP is related to the impaired circulatory functions which characterizes cirrhosis with ascites.(4) There are limitations of use of serum creatinine (SCr) to identify patients with AKI. Besides being a marker of renal function more than kidney injury, $\mathrm{SCr}$ may be inaccurate in cirrhotic patients because of their hypervolemic state, low muscular mass and decreased hepatic production of creatinine.(5) Several promising urinary biomarkers, including neutrophil gelatinaseassociated lipocalin (NGAL)(6), has been developed to aid in early diagnosis of acute kidney injury (AKI) have been discovered, facilitating the early diagnosis of AKI.(7) Neutrophil gelatinase-associated lipocalin (NGAL) is a protein released by the injured kidney tubular cells. Urinary NGAL (uNGAL) levels rise exponentially early in the course of AKI, prior to serum creatinine elevation. Increasing evidence also suggests that high levels of uNGAL can be used separately to predict clinical outcomes, including deaths in the short term. The aim of our study was to investigate the diagnostic and prognostic role of uNGAL in SBP related AKI in patients who have liver cirrhosis.

\section{Subjects and methods:}

The study was conducted on a total of 60 patients; group (1) included 30 patients with liver cirrhosis and SBP, group (2) 30 patients with liver cirrhosis without SBP as a control 
group. Patients were admitted to the hepatobiliary unit at Alexandria Main University Hospital. Informed written consent was obtained from all patients.

Exclusion criteria were:(1)Patients with chronic kidney disease more than 3 months; (2)Patients with liver or renal transplantation;(3) Patients with hepatocellular carcinoma;(4)Patient with other sources of infection (especially urinary tract infection); (5)Patient on current or recent history of nephrotoxic drugs. A detailed history was taken from all patients on admission as regards demographic data, medical history, drug history and complete physical examination. Standard laboratory tests were measured including liver function tests, renal function tests and serum electrolytes. An ultrasound abdominal examination was done to confirm normality of both kidneys, to detect ascites, and to confirm liver cirrhosis. Diagnostic paracentesis for ascetic fluid analysis and culture was done on admission. Afresh urine sample was collected from all patients on admission for measurements of uNGAL. Empirical Antibiotic therapy (intravenous cefotaxime $2 \mathrm{~g} / 8$ hours for 5days) was started in patients with SBP and modified according to culture and sensitivity.(8) AKI was determined by an increase in serum creatinine( $\mathrm{SCr}$ ) by $\geq 0.3 \mathrm{mg} / \mathrm{dL}$ or by $\geq 50 \%$ from baseline sCr.(9) Baseline $\mathrm{SCr}$ was defined as the value of $\mathrm{SCr}$ obtained in the previous 3 months. In patients with more than one value within the previous 3 months, the value closest to the admission time to the hospital was used in patients without a previous $\mathrm{SCr}$ value, the $\mathrm{SCr}$ on admission was used as a baseline.(10) "Transient AKI" was defined as a return to baseline SCr within 3 days post-AKI and "Persistent AKI" was defined as renal dysfunction without recovery within 3 days but less than 3 months. (11)The patients were followed for three months.

\section{Statistical analysis:}

Data were expressed in the form of mean \pm standard deviation (SD), or proportions as appropriate. Comparing means was done using the non-parametric Mann-Whitney Utest for non-normally distributed quantitative variables. The one-way ANOVA test was used for comparing groups. Comparison between proportions was determined by the Chi square ( $\square 2$ ) test or Fisher's Exact test (FET). We used multivariate logistic regression to estimate the role of baseline parameters in predicting 3 months mortality. Statistical significance was assessed at $\mathrm{P}<0.05$. All calculated $\mathrm{P}$ values were two-tailed.

\section{Results:}

The baseline clinicolaboratory data of the study population are showed in table 1 . The study included thirty patients with ascites and SBP and thirty patients with ascites without SBP as a control group. Our study showed that AKI was identified in twenty-one patients $(70 \%)$ in group 1 during hospitalization, (table 1).

AKI was present at diagnosis of SBP in 9 patients (42.9\%), in the remaining 12 patients, AKI developed during day 1-2 after diagnosis of SBP in five $(23.8 \%)$ patients, and in seven $(33.3 \%)$ patients after 48 hours, (table 1$)$.

Patients with AKI had significantly higher mean uNGAL levels compared to both patients without AKI and the control group ( $7.55 \pm 3.20$ vs. $2.09 \pm 1.31 \mathrm{ng} / \mathrm{ml}, \mathrm{P}<0.001$, and 7.55 \pm 3.20 vs. $1.23 \pm 0.78 \mathrm{ng} / \mathrm{ml}, \mathrm{P}<0.001$ respectively), while there was no significant difference between the mean value of uNGAL in patients without AKI in group 1 and the control group ( $\mathrm{P}=0.083)$ (table 2$)$.

There was a positive correlation between levels of uNGAL at the diagnosis of SBP and serum creatinine (figure1).

Receiver operator characteristic curve (ROC) analysis was performed to test the ability of uNGAL to diagnose AKI in cirrhotic patients with SBP. At a cut-off level of $\geq 3.56$ $\mathrm{ng} / \mathrm{ml}$, uNGAL could predict the occurrence of AKI with a sensitivity of $85.71 \%$ and a specificity of $88.89 \%$ and with a positive predictive value of $94.7 \%$ and a negative predictive value of $72.7 \%$ (area under the curve $=0.947, \mathrm{P}<0.001,95 \%$ CI: 0.87-1), (figure 2).

At the end of 3-months follow up, among the 30 patients with ascites and SBP, 22 patients $(73.3 \%)$ were alive (13 patients with $\mathrm{AKI}$ and 9 patients without AKI), while 8 patients $(26.7 \%)$ had died. The mean value of UNGAL in patients who had died was significantly higher than its mean value in alive patients $(9.90 \pm 2.21$ vs. $4.46 \pm 3.07 \mathrm{ng} / \mathrm{ml}, \mathrm{P}=0.001)$, (Table 3).

Out of 13 patients with AKI who survived, in 5 patients $(38.5 \%)$, AKI was persistent ( i.e. AKI extended beyond 3 days after the initial diagnosis of SBP and AKI)and the remaining 8 patients $(61.5 \%)$ had transient AKI (i.e. resolved within 3 days of diagnosis of SBP and AKI).The mean value of UNGAL was significantly higher in patients with persistent AKI compared to patients with transient AKI (8.88 \pm 1.51 vs. $4.37 \pm 1.95 \mathrm{ng} / \mathrm{ml}, \mathrm{P}=0.005)$, (Table 3 )

In multivariate analysis both the model of end-stage liver disease-sodium (MELD-Na) and uNGAL level at diagnosis of SBP were an independent predictor of 3 months mortality (Table 4)

\section{Discussion:}

Among cirrhotic patients, spontaneous bacterial peritonitis is a leading cause of AKI which in turn, is associated with high mortality and hospitalization. UNGAL is a recent marker for early diagnosis of AKI. Our current study was performed on patients with cirrhosis and SBP, this condition is a leading cause of severe kidney impairment. We found that in group 1 patient with SBP, $70 \%$ has AKI during hospitalization, and $42.9 \%$ already have AKI at diagnosis of SBP, and the remaining varied from 1-2 days of admission. Follo et al(1994)(12) studied the incidence of AKI in SBP and demonstrated that $30 \%$ of SBP presented with AKI. Coral GP (2003)(13) demonstrated that the occurrence of AKI in SBP was ( 55.9\%), out of 114 patients with SBP 61 patients developed AKI. The higher incidence of AKI in Coral GP et al explained that the previous literature did not consider cases of transient AKI as cases of AKI. The higher number of AKI cases in our study may be due to the delayed presentation of the patients. Our study showed that AKI was associated with higher levels of uNGAL. Patients who have AKI in group 1, compared to patient without AKI, had much higher levels of uNGAL. uNGAL level positively correlates with serum creatinine level, uNGAL was elevated one to two days prior to the elevation of serum creatinine. The ROC analysis showed that at a cut off value $>3.56 \mathrm{ng} / \mathrm{ml}$, uNGAL could 
diagnose AKI in cirrhotic patients with SBP (AUC 0.947, $\mathrm{p}<0.001)$ with sensitivity of $85.71 \%$, and specificity of $88.89 \%$.Verna EC et al (2012)(14)in their study of 180 patients hospitalized with variable cirrhosis complications, they examined a single urinary sample on admission for measurement of uNGAL and uNGAL/urinary creatinine ratio. They detected that AKI patients showed a higher uNGAL levels. Fagundes C et al (2012) (15) studied uNGALin different cases of renal impairment among a cohort of cirrhosis. Only 35\% of cases had variable degrees of kidney dysfunction. Patients with renal impairment had significantly higher uNGAL levels in comparisons with patients with / without ascites. Unlike our study, Alhaddad OM et al (2015)(16) studied the role of NGAL as a biomarker of kidney function in hepatitis $\mathrm{C}$ related advanced chronic liver disease. The study conducted on two groups of patients. First group $(\mathrm{n}=35)$ included cases with GFR $<60 \mathrm{~mL} / \mathrm{m}$, and 2 nd group $(n=65)$ included patients with GFR $\geq$ $60 \mathrm{~mL} / \mathrm{m}$. They demonstrated that uNGAL wasn't different among the two groups contrary to plasma NGAL.

The newly discovered biomarkers have the ability to detect AKI early. However, the development of biomarkers to predict the outcome of AKI is also needed. This is clinically important because it may prevent delaying the patient management among those cases with a higher probability of developing persistent AKI.The result of our study showed that a single measurement of UNGAL at detection of SBP may be helpful for the prediction of the AKI outcome. It also showed that uNGAL was higher in patients with persistent AKI compared to patients with transient AKI.

These were in agreement with Barreto $\mathrm{R}$ et al (2014)(17)in their study. They investigated cirrhotic patients with bacterial infection. They showed that persistent AKI was associated with higher UNGAL levels at diagnosis of infection in comparison to transient AKI. They explained this finding by that persistent AKI leads to greater activation of vasoactive system compared to transient AKI.Yang $\mathrm{HN}$ et al (2010)(18)where they enrolled heterogeneous group of hospitalized patients with AKI demonstrated that initial measurement of uNGAL was identified as a predictor of persistent loss of renal function in a patient with AKI. The patients were assessed 1 month after enrollment in the study, persistent loss of kidney function occurred in $(35 \%)$ of surviving patients. Yang $\mathrm{HN}$ et $\mathrm{al}(18)$ also reported appositive correlation between serum creatinine and level of uNGAL. Another interesting figure in the current work was that UNGAL level measured concurrently with diagnosis of SBP had an important association with 3months deaths. In multivariate analysis uNGAL level and baseline, MELD-Na score at admission of patients with cirrhosis and SBP could independently predict the 3 months mortality, ROC curve showed (AUC,0.92, sensitivity 87.5,specificity 77.72).This goes with the results of Barreto R et al (2014) (17)studied the role of UNGAL in the prediction of 3 months mortality in cirrhotic patients with a bacterial infection. The study reported that MELD score at baseline, serum sodium and uNGAL were predictors of 3 months death rate in patients with cirrhosis and bacterial infection. Barreto $\mathrm{R}$ et al measured uNGAL level at diagnosis and also at day 3 and 7 post-diagnosis. Barreto could not explain the uNGAL prognostic role in a cirrhotic patient with infection and suppose that it more likely related to its power to predict the persistent AKI, a condition had an impact on survival. Verna EC et al (14) reported that a single measurement of uNGAL independently predicted short term mortality in patients with AKI in cirrhotic patients adjusted for age and serum creatinine. De-Geus et al (19) a prospective cohort study on a heterogeneous group of patients admitted to ICU with different etiology and timing of AKI studied the predictive value of plasma and urinary NGAL in patients with AKI and demonstrated that UNGAL level was a good predictor to severe AKI but against with us UNGAL have minor role in predicting in-hospital mortality. De-geus reported also that serial measurement of uNGAL did not provide additional information for the prediction of AKI. In our study, we confirmed that cirrhotic patients with SBP that develop AKI had a significantly higher 90-day mortality rate than the nonAKI patients despite half of AKI episodes were transient AKI. To conclude, from the current study, we conclude that in cirrhotic patients with SBP, measuring the UNGAL at SBP diagnosis can diagnose AKI, and can predict the clinical outcome as progression of AKI during hospitalization and the 3 -month mortality. If we could confirmed our findings in a larger scale studies, the uNGAL may act as an important diagnostic marker in guiding clinical management of cirrhosis with SBP patients 
Table1. Baseline clinicolaboratory data of the study cohort:

\begin{tabular}{|c|c|c|c|}
\hline & Patients with SBP $(n=30)$ & Control $(n=30)$ & $\mathbf{P}$ \\
\hline Male sex: $n(\%)$ & $21(70)$ & $22(73.3)$ & $>0.05$ \\
\hline Age (years) & $53.37 \pm 7.46$ & $51.8 \pm 4.31$ & 0.320 \\
\hline \multicolumn{4}{|l|}{ Etiology of cirrhosis: } \\
\hline - BCS & $1(3.3)$ & $0(0.0)$ & \multirow{4}{*}{0.661} \\
\hline - Cryptogenic & $3(10.0)$ & $1(3.3)$ & \\
\hline - HBV & $2(6.7)$ & $3(10.0)$ & \\
\hline - $\mathrm{HCV}$ & $24(80.0)$ & $26(87.7)$ & \\
\hline HB (g/dl) & $10.03 \pm 1.02$ & $10.71 \pm 1.29$ & 0.028 \\
\hline WBCs $x 10^{3} / \mathrm{ccm}$ & $11.65 \pm 5.60$ & $4.9 \pm 0.88$ & $<0.001$ \\
\hline Platelets $\left(\mathrm{x10}^{3}\right) / \mathrm{ccm}$ & $88.27 \pm 39.98$ & $113.17 \pm 36.94$ & 0.004 \\
\hline INR & $1.84 \pm 0.42$ & $1.51 \pm 0.21$ & $<0.001$ \\
\hline PA ( \%) & $40.63 \pm 10.73$ & $50.80 \pm 9.73$ & $<0.001$ \\
\hline Creatinine (mg/dl) & $1.5 \pm 0.94$ & $0.66 \pm 0.14$ & $<0.001$ \\
\hline AKI present: $n(\%)$ & $21(70.0)$ & - & - \\
\hline \multicolumn{4}{|c|}{ Onset of AKI at SBP diagnosis $(n=21), n(\%)$} \\
\hline On admission & $9(42.9)$ & - & - \\
\hline 24-48 h & $5(23.8)$ & - & - \\
\hline$>48 \mathrm{hrs}$ of & $7(33.3)$ & - & - \\
\hline
\end{tabular}

SBP: Spontaneous bacterial peritonitis; HCV: Hepatitis C virus; HBV: Hepatitis B virus; AKI: Acute kidney injury; INR: International normalization ratio; PA: Prothrombin activity; Hrs: hours; Hb: Hemoglobin; WBCs: White blood cells; BCS: Budd-Chiari syndrome

Table2. Relationship between UNGAL and presence of AKI in the studied groups

\begin{tabular}{|c|c|c|c|c|c|}
\hline \multirow[b]{2}{*}{ uNGAL(ng/ml) } & \multicolumn{2}{|c|}{ Patients with SBP $(n=30)$} & \multirow[t]{2}{*}{ Control $(n=30)$} & \multirow[t]{2}{*}{$\mathrm{H}$} & \multirow[t]{2}{*}{$\mathrm{P}$} \\
\hline & $\begin{array}{l}\text { without AKI } \\
(\mathbf{n}=9)\end{array}$ & $\begin{array}{l}\text { With AKI } \\
(\mathbf{n}=21)\end{array}$ & & & \\
\hline Min. - Max. & $0.82-4.35$ & $2.30-12.70$ & $0.12-3.35$ & \multirow{3}{*}{39.226} & \multirow{3}{*}{$<0.001$} \\
\hline Mean \pm SD. & $2.09 \pm 1.31$ & $7.55 \pm 3.20$ & $1.23 \pm 0.78$ & & \\
\hline Median & 2.10 & 8.50 & 0.91 & & \\
\hline
\end{tabular}

$\mathrm{H}, \mathrm{p}: \mathrm{H}$ and $\mathrm{p}$ values for Kruskal Wallis test, Significance between groups was done using Mann Whitney test p1: p-value for comparing between patient without AKI and with AKI.

p2: p-value for comparing patient without AKI and control group.

p3: p-value for comparing patients with AKI and control group.

SBP: Spontaneous bacterial peritonitis; AKI: Acute kidney injury; uNGAL: urinary neutrophil gelatinase-associated lipodellin; Min: minimum; Max: Maximum; SD: Standard deviation 
Table3. Comparisons of the values of urinary neutrophil gelatinase-associated lipocalin [uNGAL(ng/ml)] according to both survival at three months and the outcome of acute kidney injury (AKI)

\begin{tabular}{|c|c|c|c|c|c|}
\hline & \multicolumn{5}{|c|}{ uNGAL(ng/ml) } \\
\hline & \multicolumn{3}{|c|}{ Three months survival: $n(\%)$} & $\mathbf{U}^{*}$ & $\mathbf{P}$ \\
\hline Alive: 22 (73.3) & $0.82-10.50$ & $4.46 \pm 3.07$ & 3.68 & \multirow{2}{*}{14.0} & \multirow{2}{*}{0.001} \\
\hline Dead: $8(26.7)$ & $5.0-12.7$ & $9.90 \pm 2.21$ & 10.32 & & \\
\hline \multicolumn{6}{|l|}{ Outcome of AKI: $n(\%)$} \\
\hline Transient: 8 (61.5) & $2.30-8.50$ & $4.37 \pm 1.95$ & 4.15 & \multirow{2}{*}{1.00} & \multirow{2}{*}{0.005} \\
\hline Persistent: 5 (38.5) & $6.50-10.50$ & $8.88 \pm 1.51$ & 9.40 & & \\
\hline
\end{tabular}

* U values for Mann Whitney test for comparing between the two groups.

uNGAL: urinary neutrophil gelatinase-associated lipocalin; AKI: Acute kidney injury; Min: minimum; Max: Maximum; SD: Standard deviation.

Table4. Multivariate analysis logistic regression for predictors of survival at three months among patients with ascites and spontaneous bacterial peritonitis

\begin{tabular}{|c|c|c|c|c|c|c|}
\hline & & & & & $95 \%$ & \\
\hline & B & SE & Sig. & OR & & UL \\
\hline$\overline{\text { uNGAL(ng/ml) }}$ & 0.635 & 0.244 & $0.009^{*}$ & 1.887 & 1.169 & 3.046 \\
\hline MELD-Na & 0.340 & 0.136 & $0.012^{*}$ & 1.405 & 1.077 & 1.834 \\
\hline
\end{tabular}

Figure (1): Spearmen correlation between urinary neutrophil gelatinase-associated lipocalin. (uNGAL) with serum creatinine in patients with ascites and spontaneous bacterial peritonitis.

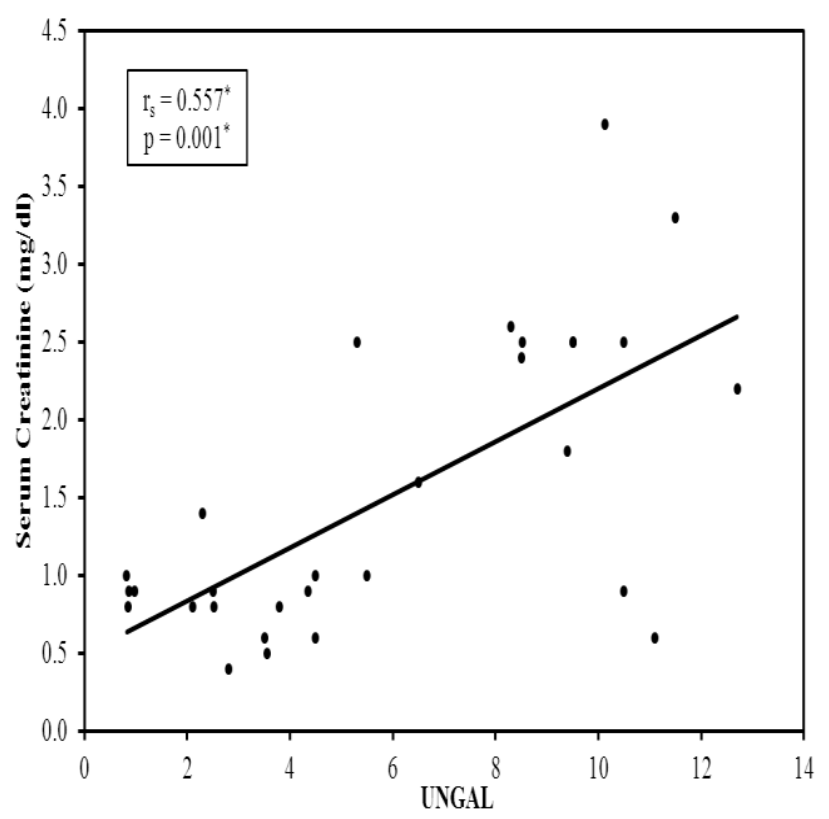

Figure (2): Receiver operator (ROC) curve for urinary neuotrophil gelatinase-associated lipocalin (uNGAL); diagnose acute kidney injury in patients with spontaneous

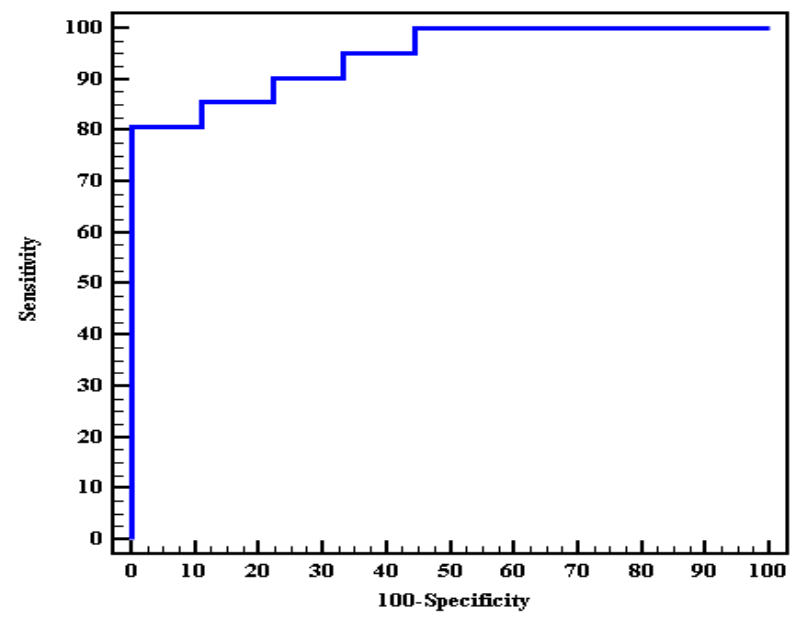

bacterial peritonitis

. AUC $=0.95, \mathrm{P}<0.001)$.

Sensitivity of $85.7 \%$ and specificity of $88.9 \%$ at a cut-off value $>3.56 \mathrm{ng} / \mathrm{ml}$, uNGAl 


\section{References}

1. Arvaniti V, D'Amico G, Fede G, Manousou P, Tsochatzis $\mathrm{E}$, Pleguezuelo $\mathrm{M}$, et al. Infections in patientswith cirrhosis increase mortality four-fold and should be used in determining prognosis. Gastroenterology. 2010; 139(4):1246-56. e5.

2. Fasolato S, Angeli P, Dallagnese L, Maresio G, Zola E, Mazza E, et al. Renal failure and bacterial infections in patients with cirrhosis: epidemiology and clinical features. Hepatology. 2007;45(1):223-9.

3. Liver EAFTSOT. EASL clinical practice guidelines on the management of ascites, spontaneous bacterial peritonitis, and hepatorenal syndrome in cirrhosis. Journal of Hepatology. 2010;53(3):397-417.

4. Tandon P, Garcia-Tsao G. Renal dysfunction is the most important independent predictor of mortality in cirrhotic patients with spontaneous bacterial peritonitis. Clin GastroenterolHepatol. 2011;9(3):260-5.

5. Slack A, Yeoman A, Wendon J: Renal dysfunction in chronic liver disease. Crit Care14:214-23, 2010.

6. Mishra J, Dent C, Tarabishi R, Mitsnefes MM, Ma Q, Kelly C, et al. Neutrophil gelatinase-associated lipocalin (NGAL) as a biomarker for acute renal injury after cardiac surgery. The Lancet. 2005;365(9466):1231-8.

7. Devarajan P. Neutrophil gelatinase-associated lipocalin - an emerging troponin for kidney injury. Nephrology Dialysis Transplantation. 2008;23(12):373743.

8. Runyon BA. Management of adult patients with ascites due to cirrhosis: update 2012. AASLD Practice Guideline, AASLD. 2012.

9. Wong F, Nadim MK, Kellum JA, Salerno F, Bellomo R, Gerbes A, et al. Working Party proposal for a revised classification system of renal dysfunction in patients with cirrhosis. Gut. 2011;60(5):702-9.

10. Angeli $\mathrm{P}$, Ginès $\mathrm{P}$, Wong $\mathrm{F}$, Bernardi $\mathrm{M}$, Boyer TD, Gerbes A, et al. Diagnosis and management of acute kidney injury in patients with cirrhosis: revised consensus recommendations of the International Club of Ascites. Gut. 2015:gutjnl-2014-308874.

11. Perinel S, Vincent F, Lautrette A, Dellamonica J, Mariat C, Zeni F,et al. Transient and Persistent Acute Kidney
Injury and the Risk of Hospital Mortality in Critically Ill Patients: Results of a Multicenter Cohort Study.Critical Care Medicin. 2015 Aug; 43(8):e269-75.

12. Follo A, Llovet JM, Navasa M, Planas R, Forns X, Francitorra A, et al. Renal impairment after spontaneous bacterial peritonitis in cirrhosis: incidence, clinical course, predictive factors and prognosis. Hepatology. 1994; 20(6):1495-501.

13. Coral GP, Alves de Mattos A. Renal impairment after spontaneous bacterial peritonitis: incidence and prognosis. Canadian Journal of Gastroenterology and Hepatology. 2003;17(3):187-90.

14. Verna EC, Brown RS, Farrand E, Pichardo EM, Forster CS, Sola-Del Valle DA, et al. Urinary neutrophil gelatinase-associated lipocalin predicts mortality and identifies acute kidney injury in cirrhosis. Digestive diseases and sciences. 2012;57(9):2362-70.

15. Fagundes C, Pépin M-N, Guevara M, Barreto R, Casals $\mathrm{G}$, Solà E, et al. Urinary neutrophil gelatinase-associated lipocalin as biomarker in the differential diagnosis ofimpairment of kidney function in cirrhosis. Journal of hepatology. 2012;57(2):267-73

16. Alhaddad OM, Alsebaey A, Amer MO, El-Said HH, Salman TAH. Neutrophil gelatinase-associated lipocalin: a new marker of renal function in C-related end stage liver disease. Gastroenterology research and practice. 2015;2015.

17. Barreto R, Elia C, Solà E, Moreira R, Ariza X, Rodríguez E, et al. Urinary neutrophil gelatinaseassociated lipocalin predicts kidney outcome and death in patients with cirrhosis and bacterialinfections. Journal of hepatology. 2014;61(1):35-42.

18. Yang HN, Boo CS, Kim M-G, Jo S-K, Cho WY, Kim H$\mathrm{K}$. Urine neutrophil gelatinase-associated lipocalin: an independent predictor of adverse outcomes in acute kidney injury. American journal of nephrology. 2010;31(6):501-9.

19. de Geus HR, Bakker J, Lesaffre EM, Le Noble JL. Neutrophil gelatinase-associated lipocalin at ICU admission predicts for acute kidney injury in adult patients. American journal of respiratory and critical care medicine. 2011;183(7):907-14. 